\title{
The impact of orthorexia nervosa on bone health
}

\author{
Luana Andreea Macovei, Ioana Brătoiu, Patricia Richter, \\ Alexandra Burlui, Anca Cardoneanu, Elena Rezuș
}

Luana Andreea Macovei - Lecturer, MD, PhD, “Grigore T. Popa” University of Medicine and Pharmacy - Iaşi, Romania, Faculty of Medicine, Department of Medical Specialties (II), Clinical Rehabilitation Hospital - Ist Rheumatology Clinic

Ioana Brătoiu - MD, PhD student, "Grigore T. Popa" University of Medicine and Pharmacy - Iaşi, Romania, Faculty of Medicine, Department of Medical Specialties (II), Clinical Rehabilitation Hospital - Ist Rheumatology Clinic

Patricia Richter - MD, PhD student, "Grigore T. Popa" University of Medicine and Pharmacy Iaşi, Romania, Faculty of Medicine, Department of Medical Specialties (II), Clinical Rehabilitation Hospital - Ist Rheumatology Clinic

Alexandra Burlui - Assistant Professor, MD, PhD, "Grigore T. Popa" University of Medicine and Pharmacy - Iaşi, Romania, Faculty of Medicine, Department of Medical Specialties (II), Clinical Rehabilitation Hospital - Ist Rheumatology Clinic

Anca Cardoneanu - Assistant Professor, MD, PhD, "Grigore T. Popa" University of Medicine and Pharmacy - Iaşi, Romania, Faculty of Medicine, Department of Medical Specialties (II), Clinical Rehabilitation Hospital - Ist Rheumatology Clinic

Elena Rezuș - Professor, MD, PhD, "Grigore T. Popa” University of Medicine and Pharmacy - Iaşi, Romania, Faculty of Medicine, Department of Medical Specialties (II), Clinical Rehabilitation Hospital - Ist Rheumatology Clinic

\section{ABSTRACT}

Dietary recommendations are meant to optimize bone health and prevent bone resorption and osteoporosis when dietary intakes do not maintain normal serum levels of calcium, phosphorus, and magnesium. The current healthy eating trend is verified in association with its effects on bone density, with particular attention to some practices: vegetarianism, lactosefree diet, alkaline diet and dietary supplements. A plant-based diet may inhibit intestinal calcium absorption, due to the impact of oxalates and phytates. Lactose was suggested to enhance calcium absorption and lactose intolerant individuals have a lower calcium intake than healthy controls. The alkaline diet, with foods associated with neutral or alkaline urine thought to be beneficial for the skeleton, is based solely on findings from patients with renal 
r.\%\% impairment, where alkaline salts, such as calcium phosphates, are released from bones in order to provide an optimal acid-base balance. Excessive vitamin and mineral consumption limits the absorption efficiency and induce detrimental structural changes in bone morphology.

\section{A healthy diet with protective effects on bone should be focused on diversification, instead of restrictions and excess.}

\section{KEYWORDS:}

Nutritional status, orthorexia nervosa, healthy diet, obsessive-compulsive disorder.

\section{INTRODUCTION}

Various dietary factors contribute to positive changes in bone turnover and there is a general belief in diet-controlled diseases. An online survey conducted in Canada in 2018 on 1108 osteoporosis patients reported the need for recommendations on nutrition in $68 \%$ of respondents(1). The role of nutrition for the osteoporosis prevention in women, in whom menopause is the main risk factor, has been shown to be based on calcium and protein intake, vitamin $\mathrm{D}$, potassium, phosphorus and other nutrients. Calcium and vitamin D insufficiency is common among women of all ages, due to inadequate nutritional habits. Only protein intake is lower in older female age groups who are prone to osteoporosis, fall risk, fractures and fragility. The menopauseinduced osteoporosis risk can be reduced by adopting a healthy lifestyle that includes adequate levels of dietary calcium, vitamin D and protein, regular physical exercise, smoking cessation and reduced alcohol use (2).

In individuals with orthorexic tendencies, dieting does not achieve solely a therapeutic goal, but rather it becomes a lifestyle. That is why orthorexia is not yet considered pathological and not recognized as a medical diagnosis in international classifications. Orthorexia, the obsession with the quality of food eaten and the tendency to incriminate some foods as dangerous, has been described in literature since 1997. In cases in which over-concern about food leads to lifestyle changes aimed at suiting eating patterns, orthorexia was linked to obsessive- compulsive disorder (3), especially because it is difficult to strictly control diet for years. Health, aesthetics and disease prevention are related to orthorexia, whereas animal welfare, politics, religion and ecology are not within its scope.

Orthorexia is considered a means of controlling body fat and body weight, with less regard to bone health. Body image issues lead many anorexic female patients in their twenties to suffer from premenopausal osteoporosis of the spine, with a rapid progression of skeletal degeneration, with a BMD loss of $2.5 \%$ per year, due to malnutrition and depressed ovarian estrogen synthesis $(4,5)$. When adhering to a restrictive diet, orthorexic individuals are not motivated by body image issues, but orthorexia nervosa could be a residual symptom of eating disorders, such as anorexia nervosa, where patients convert from food quantity to food quality, as a means of dieting in a socially acceptable manner. Malnutrition has been reported even in dietitians and a lower body mass is often related with symptoms of orthorexia nervosa (6).

Healthy eating, which discourages sodium consumption and recommends potassium intake, by focusing on fresh fruits and vegetables helps bone health and may be beneficial. However, when carried to extreme and meat safety anxiety prevails, orthorexia nervosa can lead to protein-energy malnutrition and osteoporosis. Nutritional deficiencies in orthorexia could be a major risk factor for bone fragility. 


\section{VEGETARIANISM}

A healthy diet is often seen as a vegetarian diet, due to health benefits such as a decreased risk of metabolic syndrome, ischemic heart disease or cancer, and due to concerns about possible use of hormones and antibiotics in animals. The high fat or cholesterol content of calcium-rich foods are thought to cause weight gain and cardiovascular disease. Vegetarian diets include several variants: lacto-vegetarianism, which adopts also dairy products, ovovegetarianism, with egg consumption and veganism, when flesh, dairy, egg and even honey are avoided. The extent to which an orthorexic diet could be considered healthy remains to be measured by the effects of a meat-restrictive diet, which causes nutritional deficiencies in vitamin $\mathrm{D}$, iron, calcium and zinc. Proteins provide the structural matrix of bones and account for approximately half of bone volume and about a third of bone mass. Proteins stimulate IGF-I, a hormone that increases calcium and phosphorus absorption in the gut and the rate of phosphate reabsorption from the kidney.

Even if protein consumption is important for preventing bone fractures, the excess in protein intake leads also to higher urinary calcium excretion. However, this increased calciuria is counterbalanced by the hypocalciuria triggered by meat, which is rich in phosphorus and by increased calcium absorption due to higher serum levels of insulin-like growth factor 1 . That is why highprotein diets are associated with reduced hip fractures. The protective role of foods rich in proteins has been associated with an adequate calcium intake $(7,8)$. Insufficient protein intake may be also responsible for secondary hyperparathyroidism, which causes bone loss (9).

The plant-based sources of calcium, such as legumes and nuts, contain significant amounts of calcium, but its bioavailability is dependent on the levels of phytate and oxalate present in the food. Oxalates and phytates inhibit calcium absorption. Oxalates from spinach and legumes are the strongest inhibitors of calcium absorption. Phytates from seeds form insoluble salts with calcium cations, which can be released through yeast fermentation. For that reason, high fiber foods rich in calcium, such as beans, spinach, chard and rhubarb, impair calcium intestinal absorption due to their phytate and oxalate content. The bioavailability of calcium in soy juice (tricalcium phosphate) is only $75 \%$ of that corresponding to cow's milk (10). However, dairy product intake is richer in calcium, protein, magnesium, potassium, zinc and phosphorus than any other food. By way of comparison, 3 to 4 portions of dairy are the equivalent of up to 24 servings of vegetables or 48 servings of grain foods (2).

The recommended moderate intake of meat beneficial for bone health could be provided by the Mediterranean diet, which is characterized by the intake of vegetables, legumes, fruits, fish, unrefined cereals, unsaturated lipids (olive oil), dairy products (cheese and yogurt), with small portions of meat and alcohol (red wine) (11).

\section{LACTOSE-FREE DIET}

The lactose-free diet was intended to provide an optimal calcium intake in individuals with lactose intolerance. However, avoiding dairy products, due to abdominal symptoms postconsumption, is linked to a recent trend in healthy lifestyle, the lactose-free and low-fat movement.

It has been reported that in a group of lactose intolerant adolescents there was a lower $\mathrm{Ca}$ and Vitamin D intake than in the control group, even if the lactose-free cow's milk has all the properties of regular cow's milk, including the presence of bioactive components such as the insulin-like growth factor 1, that may facilitate bone growth (12). This may be explained by the fact that lactose intolerant individuals tend to consume less dairy products than the controls but also by the beneficial role of lactose in calcium absorption, as it has also been shown by the comparative study of lactose-containing formula and lactose-free infant formulas, with a fractional and total calcium absorption significantly higher in the presence of lactose (13). Therefore, lactose is thought to stimulate mineral and especially calcium absorption. 


\section{ALKALINE DIET}

The deterioration of bone integrity was first observed in patients with severe chronic renal failure who also have a low urinary $\mathrm{pH}$. It was suggested that alkaline salts, in the form of calcium phosphates, are released from bones so that the systemic $\mathrm{pH}$ could be preserved. The acidity retained in the body could be neutralized by bicarbonates (calcium bicarbonate) coming from the dissolution of carbonate impurities found in hydroxyapatite crystals. It is highly doubtful that acid neutralization is induced via bone demineralization, due to the fact that it would take up to 8 years and about $50 \%$ of bone mineral density to achieve that (14).

In acidity related to renal insufficiency, bone demineralization is caused by renal mechanisms instead of nutritional factors, such as calcium intestinal absorption. Acidosis inhibits the renal tubular reabsorption of calcium, leading to an increased urinary calcium excretion and hence to bone resorption.

Animal experiments dating back in the nineteenth century have shown that, when eating meat, the alkaline urine of rabbits became clear and acidic, like the urine of carnivores. The degree of urinary acidity varies according to the type of consumed foods. This well documented scientific fact gave rise to the alkaline diet craze, which incriminates the Western diet, the consumption of meat, dairy products and cereals and promotes foods rich in alkaline potassium salts, such as fruits and vegetables, which might have, among other benefits, a protective effect against bone resorption and osteoporosis. Nevertheless, the human body, whose digestive system is supported by hydrochloric acid, has coping mechanisms capable of neutralizing the excess of acidity, with the result of an increased release of carbon dioxide, which is then eliminated by the respiratory system. The renal system regulates the acid-base balance through the renal tubular system. Even if the kidneys play an essential role in maintaining an extracellular $\mathrm{pH}$ of 7.35 , no association has been found between osteoporosis and age- related renal diseases (14). So far, there is no evidence that bone instead of kidney could maintain acid-base homeostasis.

The Framingham Osteoporosis Study has also shown that there is no relationship between nutritionally induced variations of urinary acid excretion and calcium balance, bone metabolism and the risk of osteoporotic fractures, by reporting that the subjects with the highest protein intakes in this study had the highest bone mineral density. The Framingham Offspring Study showed that yoghurt intake showed a protective effect against hip fracture, while no other dairy groups showed a similar positive impact (9).

\section{VITAMINS AND MINERALS IN EXCESS}

Plant extracts or synthetic vitamin and mineral supplementation in the form of powders, liquids, tablets and capsules have become an obsession and are thought to complement a perfect diet.

Calcium and vitamin D supplementation associated with long term use of alkaline salts raise concern about toxicity and the risk of vascular calcification, due to calcium deposition in soft tissues. Calcium supplements, particularly when used in the absence of vitamin D, were linked to an increased risk of myocardial infarction. High calcium intakes can also impair zinc absorption. Potential adverse effects of excessive calcium intake are hypercalcemia, vascular or other soft tissue calcification, kidney stones, prostate cancer, interactions with other minerals such as iron and zinc, and constipation. For vitamin D, excessive intake may lead to vitamin D intoxication, marked by hypercalcemia and hypercalciuria.

Excessive zinc consumption limits the size of hydroxyapatite crystals. Chronically high phosphorus intake may contribute to elevated plasma phosphate, which can lead to hyperparathyroidism and subsequent high bone turnover, and is also associated with vascular calcifications and cardiovascular death, particularly in people with chronic kidney disease (15). 
Excessive vitamin A intake through supplementation have been associated with low body mass density and fracture risk, and excessive vitamin A intakes are associated with poor bone mineralization.

In minerals such as calcium, magnesium, and zinc, absorption efficiency decreases as the nutrient intake increases. This explains why smaller doses divided throughout the day may lead to more efficient absorption than a single large dose.

Food sources of calcium pose no risk of cardiovascular events, with benefits on bone density and prevention of fractures. Therefore food sources of macronutrients and micronutrients are preferable for maintaining bone health.

Current evidence has shown that calcium and vitamin D supplements are not required in healthy individuals and in patients receiving drug treatment for osteoporosis with an adequate vitamin $\mathrm{D}$ status and a normal calcium intake (16).

\section{DISCUSSION}

Sun exposure has an important role in preventing osteoporosis, as shown by data suggesting that, when comparing Scandinavian to Mediterranean populations, urban to rural dwellers and Caucasian race to other races, the latter groups are less prone to bone fracture (7). Genetic factors, gender and race determine differences in calcium retention after similar dietary calcium intakes, with black and Asian individuals retaining higher amounts of calcium than whites and men with higher intestinal calcium absorption and lower urinary calcium excretion than women.

A calcium daily intake below $400 \mathrm{mg}$ is linked to an increased risk of osteoporosis, but during menopause, calcium supplementation cannot slow down bone loss. However, various factors, including dietary patterns contribute to changes in bone turnover.

A balanced nutrition plan is more useful for preventing bone damage than for reversing the effects of bone fragility. Diet involvement in preventing osteoporosis is mainly associated with calcium intake, which maintains bone density, but also with nutrients containing vitamin $\mathrm{D}$, protein, fat, magnesium, phosphorus, zinc, iron, copper, manganese, vitamin $\mathrm{C}$, vitamin $\mathrm{K}$, the $\mathrm{B}$ vitamins, vitamin $\mathrm{A}$, potassium and dietary estrogen (phyto-oestrogens), even if the latter has little effect on bone metabolism. They are found in dairy, meat, beans, fruits and vegetables, nuts and seeds, fish and seafood. Tobacco and alcohol use, coffee and tea drinking, diet drinks, mineral water, eating according to religious practices such as halal, kosher or fasting are beyond the scope of our interest.

Foods rich in calcium are milk and dairy products (yogurt, cheese, ice cream), bread, some species of fish (sardines, anchovies), foods fortified with calcium (soy and almond milk) or fruits and vegetables (figs, almonds, hazelnuts, kale, beans, sesame) $(7,8)$. Vitamin $\mathrm{D}$ is present in meat, fatty fish, liver, mushrooms, eggs and foods fortified with vitamin D (dairy products and cereals) $(7,17)$. Dietary sources of vitamin D are less known to the public at large. Minerals such as potassium, magnesium, vitamin $\mathrm{C}$ and vitamin $\mathrm{K}$ are found in fruits and vegetables (18). A safe moderate protein intake (meat, fish) also improves bone health through amino acids and trace minerals, such as copper, manganese and zinc, which are important for the synthesis of the bone matrix and bone growth.

Dietary calcium compensates for calcium demand or loss through skin, hair, nails, sweat digestive secretions and urine. A calcium daily intake below $400 \mathrm{mg}$ is linked to an increased risk of osteoporosis, but during menopause calcium supplementation cannot slow down bone loss, which is, however, slower in overweight women, who have a higher calcium absorption efficiency than their slimmer counterparts, due to nutritional abundance, a more intense mechanical stimulus on bones and higher estrogen levels, as estrogen is also involved in the pathogenesis of obesity $(19,20)$. That is why 
r.\%\%

dietary interventions in osteoporosis are not aimed at reducing calorie intake and do not encourage dieting (21).

Beyond their ingredients, foods release their nutrients based on how they were cultivated, raised and cooked. The current practice of adding lime to acidic soils leads to a decreased magnesium content in plants. Unleavened bread without yeast may cause zinc deficiency.
Osteoporosis and osteopenia attributable to poor nutrition have been associated with nutrient deficiencies that occur during childhood and teenage years and are apparent in bone health only in the fifth or sixth decade of life (16). It is prudent that parents do not expose their children to the current vegan mania. Moreover, higher intake of calciumrich foods during pregnancy tends to result in a higher bone mineral density of children at later ages (2).

\section{CONCLUSIONS}

Healthy eating should not be shaped by the marketing strategies of food companies. Any food restriction can easily turn into food deprivation and malnutrition, with severe damage to bone quality and mental health. Current research remains inconclusive as to which dietary pattern is most beneficial for bone health and the excess of some vitamins and minerals has also been shown to be harmful for bone health. Therefore, healthy eating should be understood as a guideline for a nonrestrictive and non-repetitive diet, rather than a lifestyle and a short-lived trend. Physical exercise and an active lifestyle should complement any dietary approach.

\section{ACKNOWLEDGEMENTS AND DISCLOSURES}

The authors state that there are no declared conflicts of interest regarding this paper.

\section{REFERENCES}

1. Morin SN, Djekic-Ivankovic M, Funnell L, et al. Patient engagement in clinical guidelines development: input from > 1000 members of the Canadian Osteoporosis Patient Network. Osteoporos Int. 2020 May;31(5):867-874. 2. Rizzoli R, Bischoff-Ferrari H, Dawson-Hughes B, Weaver C. Nutrition and bone health in women after the menopause. Womens Health (Lond). 2014 Nov;10(6):599-608.

3. Mitrofanova E, Mulrooney H, Petroczi A. Assessing psychological and nutritional impact of suspected orthorexia nervosa: a cross-sectional pilot study. J Human Nutr Diet. 2020 Jul 30.

4. Acosta L. Anorexia Nervosa and Premenopausal Osteoporosis. Top Clin Nutr. 2017 Jul 1;32(3):252-65.

5. Robinson L, Micali N, Misra M. Eating disorders and bone metabolism in women. Curr Opin Pediatr. 2017 Aug;29(4):488-496.

6. Głąbska D, Rahelić V, Guzek D, et al. Dietary Health-Related Risk Factors for Women in the Polish and Croatian Population Based on the Nutritional Behaviors of Junior Health Professionals. Sustainability. 2019 Jan;11(18):5073.

7. Prentice A. Diet, nutrition and the prevention of osteoporosis. Public Health Nutr. 2004 Feb;7(1a):227-43.

8. Ilich JZ, Kelly OJ, Kim Y, Spicer MT. Low-grade chronic inflammation perpetuated by modern diet as a promoter of obesity and osteoporosis. Arh Hig Rada Toksikol. 2014 Jun 1;65(2):139-48.

9. Tucker KL, Hannan MT, Kiel DP. The acid-base hypothesis: diet and bone in the Framingham Osteoporosis Study. Eur J Nutr. 2001 Oct 1;40(5):231-7.

10. Lemale J, Mas E, Jung C, et al. Hepatology FS. Vegan diet in children and adolescents. Recommendations from the French-speaking Pediatric Hepatology, Gastroenterology and Nutrition Group (GFHGNP). Arch Pediatr. 2019 Oct 1;26(7):442-50.

11. Volpe R, Sotis G, Gavita R, et al. Healthy Diet to Prevent Cardiovascular Diseases and Osteoporosis. High Blood Press Cardiovasc Prev. 2012 Jun 1;19(2):65-71.

12. Baldan A, Tagliati S, Saccomandi D, et al. Assessment of Lactose-Free Diet on the Phalangeal Bone Mineral Status in Italian Adolescents Affected by Adult-Type Hypolactasia. Nutrients. 2018 May 1;10(5):558.

13. Abrams SA, Griffin IJ, Davila PM. Calcium and zinc absorption from lactose-containing and lactose-free infant formulas. Am J Clin Nutr. 2002 Aug;76(2):442-6.

14. Bonjour JP. Nutritional disturbance in acid-base balance and osteoporosis: a hypothesis that disregards the essential homeostatic role of the kidney. Br J Nutr. 2013 Oct;110(7):1168-77. 


\section{(15)}

15. Weaver CM, Gallant KM. Chapter 14. Nutrition. In: Burr DB, Allen MR (eds.). Basic and applied bone biology. Academic Press, San Diego. 2014:283-97.

16. Chen LR, Hou PH, Chen KH. Nutritional Support and Physical Modalities for People with Osteoporosis: Current Opinion. Nutrients. 2019 Nov 20;11(12):2848.

17. Shi Y, Zhan Y, Chen Y, Jiang Y. Effects of dairy products on bone mineral density in healthy postmenopausal women: a systematic review and meta-analysis of randomized controlled trials. Arch Osteoporos. 2020 Dec;15(1):1-8.

18. Levis S, Lagari VS. The role of diet in osteoporosis prevention and management. Curr Osteoporos Rep. 2012 Dec $1 ; 10(4): 296-302$.

19. Williams GP. The role of oestrogen in the pathogenesis of obesity, type 2 diabetes, breast cancer and prostate disease. Eur J Cancer Prev. 2010 Jul;19(4):256-71.

20. Surała O, Malczewska-Lenczowska J, Sadowska D, et al.Traits of Orthorexia Nervosa and the Determinants of These Behaviors in Elite Athletes. Nutrients. 2020 Sep 2;12(9):2683.

21. Noel SE, Mangano KM, Mattei J, et al. Dietary Approaches to Stop Hypertension, Mediterranean, and Alternative Healthy Eating indices are associated with bone health among Puerto Rican adults from the Boston Puerto Rican Osteoporosis Study. Am J Clin Nutr. 2020 Jun 1;111(6):1267-1277.

\section{Correspondence:}

Luana Andreea Macovei,

Lecturer, MD, PhD, “Grigore T. Popa” University of Medicine and Pharmacy - Iasi, Romania, Faculty of Medicine, Department of Medical Specialties (II), Clinical Rehabilitation Hospital - Ist Rheumatology Clinic, luanam77@yahoo.com

Submission: 03 may 2021 Acceptance: 10 jun 2021 